\title{
COMPACT KLEIN SURFACES OF GENUS 5 WITH A UNIQUE EXTREMAL DISC
}

\author{
GOU NAKAMURA
}

\begin{abstract}
A compact (orientable or non-orientable) surface of genus $g$ is said to be extremal if it contains an extremal disc, that is, a disc of the largest radius determined only by $g$. The present paper concerns non-orientable extremal surfaces of genus 5 . We represent the surfaces as side-pairing patterns of a hyperbolic regular 24-gon, that is, a generic fundamental region of an NEC group uniformizing each of the surfaces. We also describe the group of automorphisms of the surfaces with a unique extremal disc.
\end{abstract}

\section{INTRODUCTION}

The unit disc $\mathbb{D}=\{z \in \mathbb{C} ;|z|<1\}$ is the universal covering for a compact hyperbolic surface $S$ of genus $g$, where $g$ denotes the number of handles $(g \geq 2)$ if $S$ is orientable, or the number of cross caps $(g \geq 3)$ if $S$ is non-orientable. The hyperbolic metric on $S$ is the one induced by the hyperbolic metric $d s=$ $2|d z| /\left(1-|z|^{2}\right)$ on $\mathbb{D}$. C. Bavard showed in $[\mathrm{B}$ that the radius $r$ of a disc embedded in $S$ satisfies the inequality

$$
\cosh r \leq \frac{1}{2 \sin \frac{\pi}{6-6 \chi_{g}}},
$$

where $\chi_{g}$ denotes the Euler characteristic

$$
\chi_{g}= \begin{cases}2-2 g & (S: \text { orientable }) \\ 2-g & (S: \text { non-orientable }) .\end{cases}
$$

For each case we denote by $R_{g}$ the radius satisfying the equality in (1.1). A compact surface $S$ of genus $g$ is called an extremal surface if $S$ contains an extremal disc, a disc of radius $R_{g}$. Our concern here is non-orientable surfaces (Klein surfaces). A non-orientable extremal surface of genus $g \geq 3$ is uniformized by an NEC group whose fundamental region is a hyperbolic regular $(6 g-6)$-gon. The polygon, denoted by $P_{g}$, has angles of $2 \pi / 3$, so that three vertices project to one point on the surface. Hence the sides of $P_{g}$ project to a trivalent graph with $2 g-2$ vertices and $3 g-3$ edges on the surface. The trivalent graphs are used to obtain side-pairing patterns of $P_{g}$. For $g=3$ and 4, all non-orientable extremal surfaces of genus $g$ are presented in GN] and [N1 by showing the side-pairing patterns of $P_{g}$; their groups of automorphisms are also studied there. Furthermore it is revealed that non-orientable extremal surfaces of genus $g \geq 7$ admit a unique extremal disc (see [GN]). In the present paper we consider the case of $g=5$. Using the trivalent

Received by the editors April 16, 2012.

2010 Mathematics Subject Classification. Primary 30F50; Secondary 05C10.

This work was supported by Grant-in-Aid for Young Scientists (B) (No. 20740081), Japan Society for the Promotion of Science. 
graphs with 8 vertices and 12 edges, we give all the side-pairing patterns of a hyperbolic regular 24-gon that make non-orientable surfaces of genus 5. Furthermore we study the groups of automorphisms of non-orientable extremal surfaces of genus 5 with a unique extremal disc. The surfaces with more than one extremal disc are studied in $\mathrm{N2}$.

\section{Side-PAIRING PATTERNS}

Let $P$ be a hyperbolic regular 24-gon in $\mathbb{D}$. By identifying pairs of sides of $P$ properly, we can construct a non-orientable extremal surface $S$ of genus 5 . We shall obtain all such side-pairing patterns of $P$. Since the sides of $P$ project to a trivalent graph $G$ with 8 vertices and 12 edges on $S$, a walk on the sides of $P$ once in a certain direction corresponds to a walk on every edge of $G$ twice, which is called a closed walk on $G$. Note that we can walk on edges of $G$ twice in the same direction. Conversely, a closed walk on an arbitrary trivalent graph with 8 vertices and 12 edges corresponds to a side-pairing pattern of $P$. Then the surfaces obtained from the side-pairing patterns are necessarily non-orientable because an orientable extremal surface of genus $g$ corresponds to a hyperbolic regular $(12 g-6)$-gon (see [B] ), so that 24 is never attained for any genus $g$. Consequently, we consider all the closed walks on each of the trivalent graphs $G$.

We shall describe how to obtain all the trivalent graphs $G$. We connect 8 vertices $1,2, \ldots, 8$ as follows: First, there are three ways, (A), (B) and (C), to connect the vertex 1 to the others (Figure 1). Next we connect the vertex 2 to the others. For (A), there are two ways: 2 is connected to 3 by two edges or connected to 3 and 4 by one edge respectively. For (B), there are two ways: 2 is connected to 3 or 4 by one edge. For (C), there are three ways: 2 is connected to 3 and 4,3 and 5 , or 5 and 6 . We only repeat this process for the rest of the vertices $3, \ldots, 8$, where loops are not needed in (B); loops and double edges are not needed in (C).

We shall give an example to show how a closed walk on $G$ induces a side-pairing pattern of $P$. Figure 2 shows a trivalent graph, a closed walk on it, and a sidepairing pattern, where a line (resp. a dotted line) connecting two sides of the regular 24-gon denotes a pair with the opposite (resp. same) direction.

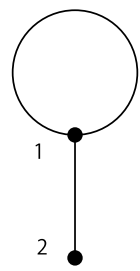

(A)

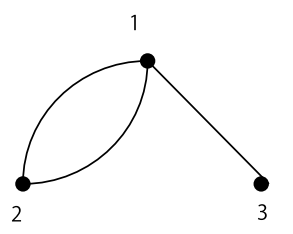

(B)

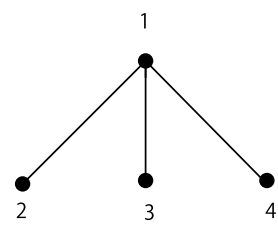

(C)

FiguRE 1. Edges connecting the vertex 1 

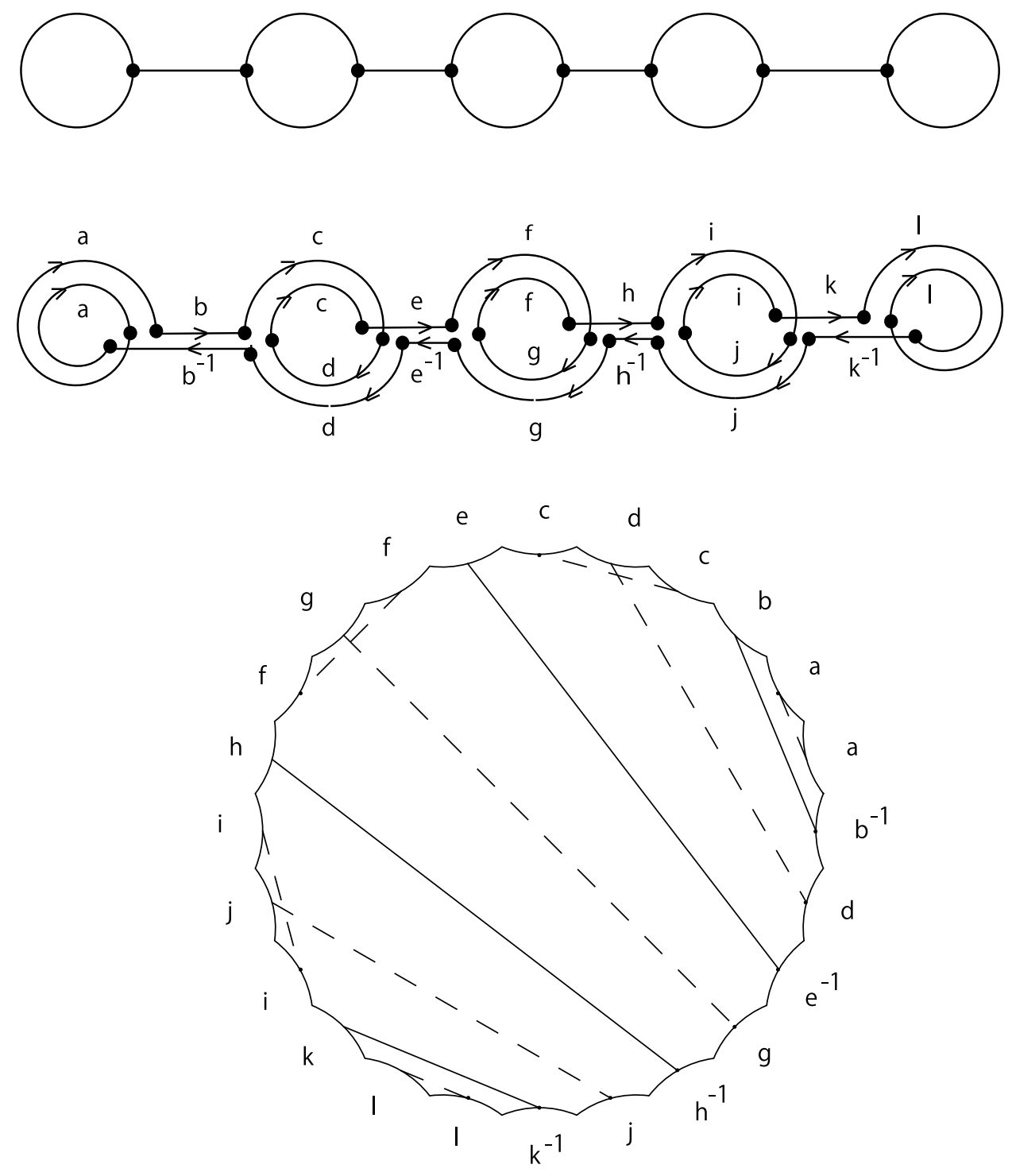

FIgURE 2. A graph, a closed walk and a corresponding side-pairing pattern

We obtained the following result via the use of a computer.

Theorem 2.1. There exist 71 trivalent graphs with 8 vertices and 12 edges (Figures 3 and 4). There exist 3627 side-pairing patterns for the regular 24-gon to be a non-orientable extremal surface of genus 5 . The surfaces obtained from these sidepairings are not isomorphic to each other.

Table 1 shows the numbers of side-pairing patterns (denoted by s.p.) derived from each of the 71 trivalent graphs $G$.

Since there are so many side-pairing patterns of the regular 24-gon, we show all of them in $\mathrm{N} 3$. 


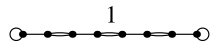<smiles>OCC=CC(O)O</smiles><smiles>OC1CC1C(O)C1CC1</smiles><smiles>OCC1CC1O</smiles>
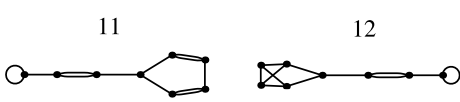<smiles>OC(O)C(O)C1CC1</smiles><smiles>OC(O)C1C=CC2C=C21</smiles><smiles>OC1C(O)C2C(O)C12</smiles>
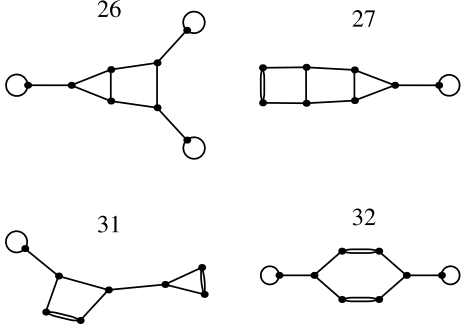<smiles>OC(O)C1C2C(O)C12</smiles>
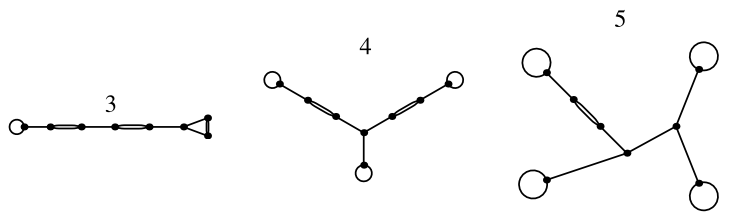<smiles></smiles><smiles>OC=CC1C2CC12</smiles><smiles>OC=CC1CCC1O</smiles>

13

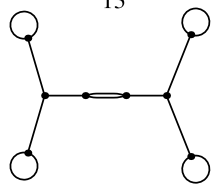<smiles>OC(O)C1C(O)C1O</smiles><smiles>OC1C2C(C3CC3)C12</smiles><smiles>OC1C(O)C1C1CC1</smiles><smiles>OC1C2C=CC=CC12</smiles><smiles></smiles><smiles>OC1CC2C1CC1C(O)C(O)C12</smiles><smiles>OC(O)C(O)C(O)O</smiles>

20<smiles>OC(O)C1CCC1O</smiles>

25<smiles>OC1C2C3C(O)C4C2C1C34</smiles>

Figure 3. Trivalent graphs with 8 vertices and 12 edges

We shall consider the group of automorphisms of non-orientable surfaces of genus 5 which admit a unique extremal disc. The surfaces admitting more than one extremal disc are studied in [N2. In the 3627 side-pairing patterns $\mathbf{1}, \mathbf{2}, \ldots, \mathbf{3 6 2 7}$, we showed that 17 of them correspond to the surfaces with more than one extremal disc [N2], which are those labeled as $684,1158,1353,1354,1356,1372,1471$, 1490, 1514, 1842, 1985, 1992, 1994, 2139, 2240, 3365, 3379. We therefore give all the groups of automorphisms of the other 3610 surfaces.

Theorem 2.2. The groups of automorphisms of the non-orientable surfaces of genus 5 with a unique extremal disc are classified as follows:

(1) $D_{3}: 803,2765,3431,3509$.

(2) $\mathbb{Z}_{3}: \mathbf{3 4 3 6 , 3 4 8 6 .}$ 

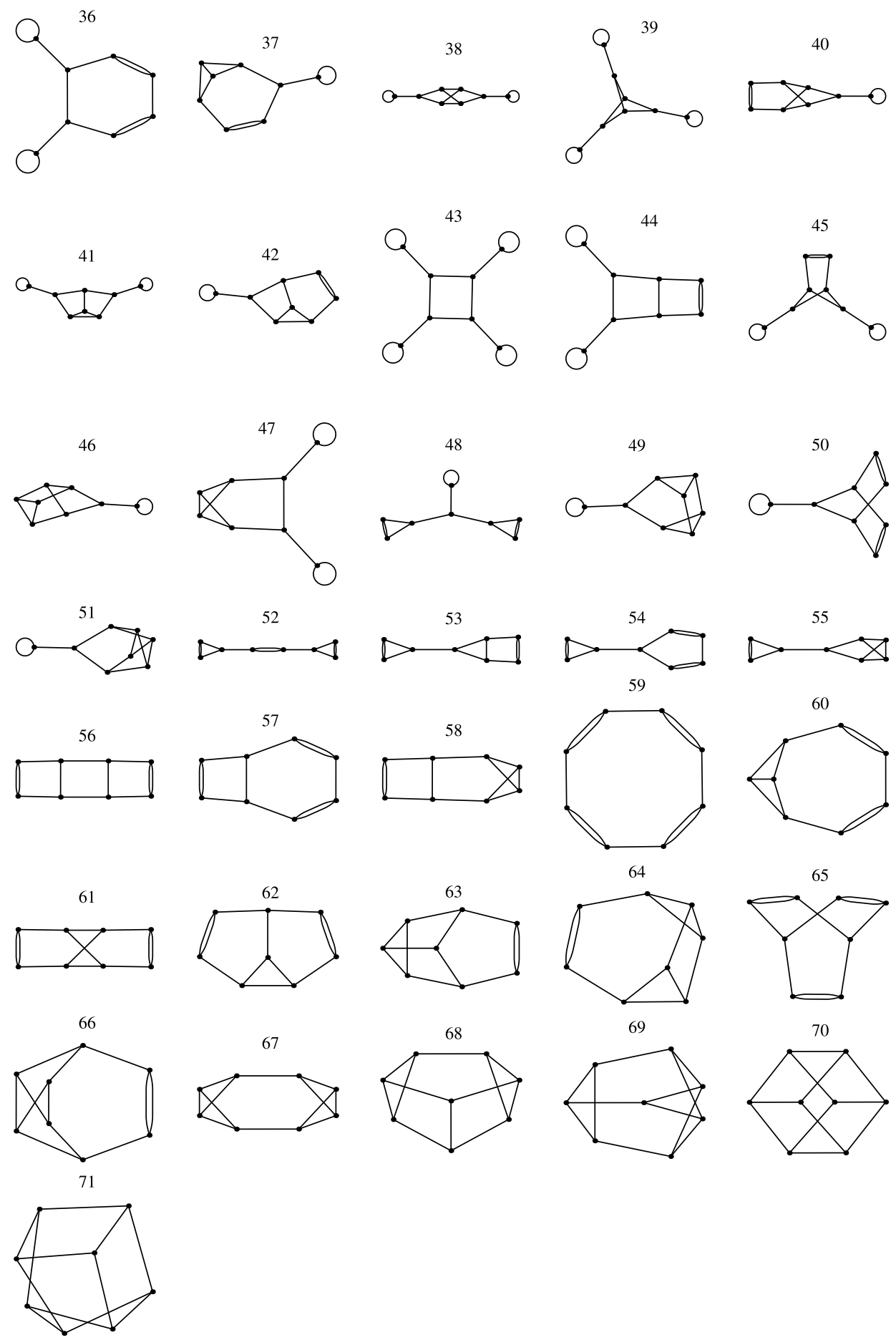

Figure 4. Trivalent graphs with 8 vertices and 12 edges 
TABLE 1. The numbers of side-pairing patterns

\begin{tabular}{|c|c|c|c|c|c|c|c|c|c|c|c|}
\hline$G$ & s.p. & $G$ & s.p. & $G$ & s.p. & $G$ & s.p. & $G$ & s.p. & $G$ & s.p. \\
\hline 1 & 3 & 13 & 1 & 25 & 26 & 37 & 88 & 49 & 110 & 61 & 40 \\
\hline 2 & 2 & 14 & 4 & 26 & 18 & 38 & 16 & 50 & 24 & 62 & 144 \\
\hline 3 & 8 & 15 & 1 & 27 & 84 & 39 & 4 & 51 & 56 & 63 & 328 \\
\hline 4 & 3 & 16 & 4 & 28 & 48 & 40 & 46 & 52 & 10 & 64 & 162 \\
\hline 5 & 2 & 17 & 8 & 29 & 34 & 41 & 60 & 53 & 52 & 65 & 15 \\
\hline 6 & 8 & 18 & 3 & 30 & 90 & 42 & 176 & 54 & 26 & 66 & 88 \\
\hline 7 & 16 & 19 & 14 & 31 & 32 & 43 & 2 & 55 & 58 & 67 & 87 \\
\hline 8 & 4 & 20 & 8 & 32 & 8 & 44 & 30 & 56 & 76 & 68 & 388 \\
\hline 9 & 24 & 21 & 7 & 33 & 18 & 45 & 13 & 57 & 62 & 69 & 137 \\
\hline 10 & 16 & 22 & 15 & 34 & 10 & 46 & 208 & 58 & 154 & 70 & 40 \\
\hline 11 & 12 & 23 & 32 & 35 & 80 & 47 & 32 & 59 & 8 & 71 & 118 \\
\hline 12 & 28 & 24 & 10 & 36 & 14 & 48 & 10 & 60 & 64 & Total & 3627 \\
\hline
\end{tabular}

(3) $\mathbb{Z}_{2}:$

$1,3,14,16,127,132,146,147,154,155,156,157,171,172,184$, $185,225,226,232,233,242,248,258,259,272,273,274,275$, $347,348,349,350,352,353,354,355,438,439,440,441,528$, 529, 530, 531, $568569,571,572,586,588,590,591,596,597$, 599, $600,682,686,687,785,790,798,799,802,846,847,848,849$, $858,860,868,879,900,905,907,909,1086,1087,1088,1089$, 1095, 1098, 1099, 1100, 1103, 1104, 1105, 1106, 1107, 1109, 1129, 1130, 1339, 1341, 1346, 1351, 1358, 1375, 1378, 1380, 1473, 1475, $1476,1479,1480,1482,1484,1486,1487,1488,1506,1508,1509$, $1510,1511,1512,1513,1566,1567,1569,1570,1571,1575,1578$, $1580,1611,1612,1613,1614,1625,1626,1627,1628,1646,1647$, 1653, 1654, 1694, 1695, 1709, 1710, 1734, 1737, 1738, 1739, 1740, $1741,1742,1743,1744,1745,1758,1764,1779,1781,1782,1783$, $1784,1785,1786,1787,1789,1790,1791,1792,1797,1798,1799$, $1800,1828,1829,1834,1839,1843,1848,1853,1883,1884,1885$, 1886 , 1952, 1953, 1960, 1962, 1963, 1965, 1967, 1972, 1984, 1995, 1997, 1999, 2004, 2009, 2010, 2012, 2013, 2015, 2016, 2068, 2070, 2071, 2072, 2074, 2077, 2078, 2080, 2090, 2093, 2098, 2099, 2100, 2101, 2102, 2106, 2115, 2117, 2119, 2120, 2125, 2131, 2144, 2199, 2205, 2237, 2245, 2249, 2253, 2256, 2260, 2261, 2263, 2264, 2480, 2483, 2487, 2488, 2504, 2507, 2518, 2519, 2540, 2543, 2547, 2548, 2564, 2567, 2578, 2579, 2635, 2637, 2639, 2640, 2643, 2644, 2646, 2648, 2650, 2651, 2652, 2654, 2707, 2709, 2720, 2728, 2733, 2735, 2746, 2754, 2756, 2758, 2760, 2761, 2762, 2763, 2764, 2793, 2794, 2796, 2797, 2831, 2833, 2836, 2841, 2847, 2849, 2852, 2857, 2863, 2879, 2897, 2898, 2901, 2911, 2914, 2919, 2920, 2925, 2927, 2933, 2940, 2944, 2971, 2980, 2981, 2992, 2996, 2997, 3009, 3010, 3030, 3031, 3050, 3066, 3123, 3139, 3144, 3171, 3176, 3197, 3201, 3204, 3207, 3212, 3218, 3228, 3259, 3263, 3266, 3276, 3279, 3280, 3289, 3294, 3299, 3300, 3304, 3313, 3328, 3329, 3331, 3332, 3360, 3382, 3387, 3388, 3389, 3390, 3404, 3428, 3449, 3450, 3451, 3452, 3454, 
$3476,3485,3489,3490,3496,3499,3500,3503,3504,3505,3508$, 3521, 3528, 3532, 3537, 3543, 3545, 3548, 3557, 3560, 3564, 3570, 3573 , 3574, 3576, 3577, 3578, 3584, 3585, 3587, 3588, 3590, 3592, 3594, 3595, 3598, 3605, 3610, 3614, 3616, 3617, 3621, 3622, 3623, 3624, 3625, 3627 (402 surfaces).

(4) $\{1\}$ : the others (3202 surfaces).

Proof. Suppose that the regular 24-gon is located in such a way that the center is the origin 0 of $\mathbb{D}$. Hence 0 corresponds to the center of the unique extremal disc. Since an automorphism $T$ of each surface fixes the center of the extremal disc, we can assume that the lift $\tilde{T}: \mathbb{D} \rightarrow \mathbb{D}$ fixes 0 . Therefore $\tilde{T}$ is a rotation around 0 or a reflection in a line passing through 0 . Considering the side-pairing patterns, we see that the side-pairing patterns in (1) admit the rotation by an angle $2 \pi / 3$ about 0 and a reflection, so that the surfaces derived from these side-pairing patterns have the group of automorphisms isomorphic to $D_{3}$, the dihedral group of order 6 . For the side-pairing patterns in (2) or in (3), we see that they admit the rotation by an angle $2 \pi / 3$ about 0 or a reflection, which implies the cyclic group of order 3 or 2 , respectively. The side-pairing patterns in (4) imply only an identity mapping. It is proved in a similar fashion that there exists no isomorphism between any two of the surfaces.

Figure 5 shows the side-pairing patterns of the regular 24-gons corresponding to the surfaces admitting $\mathbb{Z}_{3}$ or $D_{3}$ as the group of automorphisms.
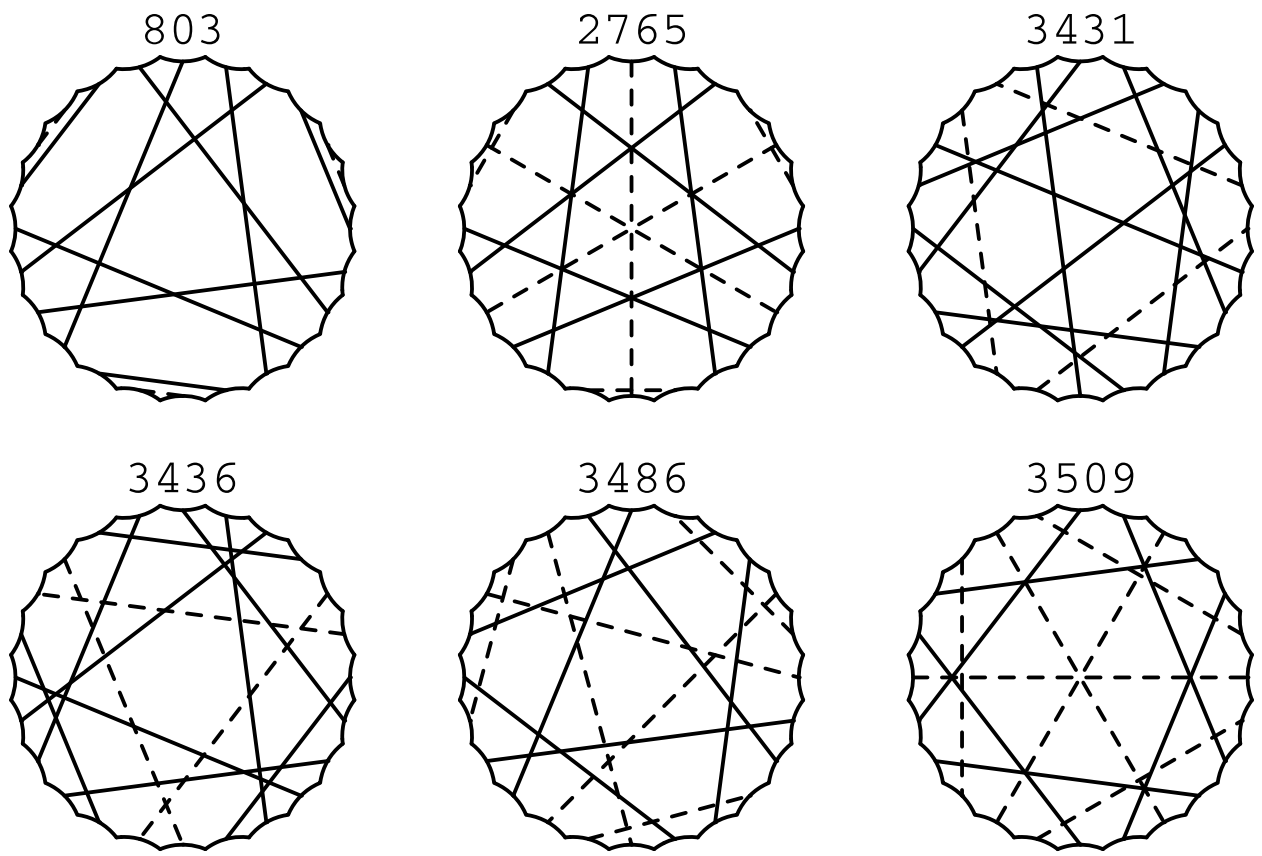

FiguRE 5. 6 side-pairing patterns 


\section{REFERENCES}

[B] Christophe Bavard, Disques extrémaux et surfaces modulaires, Ann. Fac. Sci. Toulouse Math. (6) 5 (1996), no. 2, 191-202 (French, with English and French summaries). MR1413853 (97i:30059)

[GN] Ernesto Girondo and Gou Nakamura, Compact non-orientable hyperbolic surfaces with an extremal metric disc, Conform. Geom. Dyn. 11 (2007), 29-43 (electronic), DOI 10.1090/S1088-4173-07-00157-9. MR2295996 (2007k:30087)

[JN] Troels Jørgensen and Marjatta Näätänen, Surfaces of genus 2: generic fundamental polygons, Quart. J. Math. Oxford Ser. (2) 33 (1982), no. 132, 451-461, DOI 10.1093/qmath/33.4.451. MR679814(84c:51029)

[N1] Gou Nakamura, Compact non-orientable surfaces of genus 4 with extremal metric discs, Conform. Geom. Dyn. 13 (2009), 124-135, DOI 10.1090/S1088-4173-09-00194-5. MR2497316 (2010d:30053)

[N2] Gou Nakamura, Compact non-orientable surfaces of genus 5 with extremal metric discs, Glasg. Math. J. 54 (2012), no. 2, 273-281, DOI 10.1017/S0017089511000589. MR2911368

[N3] Gou Nakamura, http://aitech.ac.jp/ nakamura/sp.html.

Science Division, Center for General Educhtion, Aichi Institute of Technology, YAKUSA-Cho, TOYOTA 470-0392, JAPAN

E-mail address: gou@aitech.ac.jp 